Experiments involving so-called elastic collisions between an atom and a cavity should allow the non-destructive detection of very small numbers of photons and the direct measurement of cavity field quantum jumps.

\title{
Measuring Photons Non-Destructively
}

An atom and a small cavity with highly reflecting walls can couple and correlate strongly to each other, resulting in phenomena which may lead to the development of new kinds of field sensors to detect photons without destroying them; the same phenomena also illustrate basic concepts of quantum mechanics. The study of these effects, at first theoretical and now entering an active experimental phase, forms a part of cavity quantum electrodynamics [1], a growing field spanning atomic physics and quantum optics with many potential applications.

\section{The Atom-Cavity "Molecule"}

Consider an atom in a cavity whose size matches exactly the wavelength of a photon the atom can emit in a transition between two of its energy levels. If the atom is initially excited and the resonator empty, a photon will be radiated within a short period of time in the cavity. Since this photon is trapped in the atom's vicinity by the reflecting cavity walls, it will be absorbed at a later time, the atom jumping back to the upper level, commencing an ideally endless cycle of photon emission and absorption processes. Remarkably, the exchanged photon also mediates a small force between the atom and the cavity walls [2], in the same way as charged particles exert a force on each other by exchanging a photon, or atoms attract each other in a molecule by trading electrons.

The molecule analogy can help us understand some basic features of the atom-cavity system. Two atoms with chemical affinity exchange an electron at a rate which depends on their distance apart. As a result, they experience a force, which either ties them together in a molecular bound state, or repels them from each other in an "antibinding" state. The same physics goes on in the atom-cavity system, albeit at a quite different energy scale. This system has two energy eigenstates, namely a binding one in which the excitation is shared symmetrically between the atom and the cavity (sum of the excited atom with no photon and the deexcited atom with one photon), and an anti-

Serge Haroche teaches and does research at the Ecole Normale Supérieure, 24, rue Lhomond, F-75005 Paris. He studied at the ENS, receiving his doctorate in 1971, and worked at the CNRS before becoming a professor of physics at the Université de P. et M. Curie, Paris, in 1975. A visitor at Stanford and Harvard, he has been a part-time professor at Yale University since 1984. He won the French Physical Society's 1983 Jean Ricard Prize, a 1992 Humbolt Award, and the Franklin Institute's 1993 Michelson Medal.

\section{S. Haroche \\ Ecole Normale Supérieure, Paris, France}

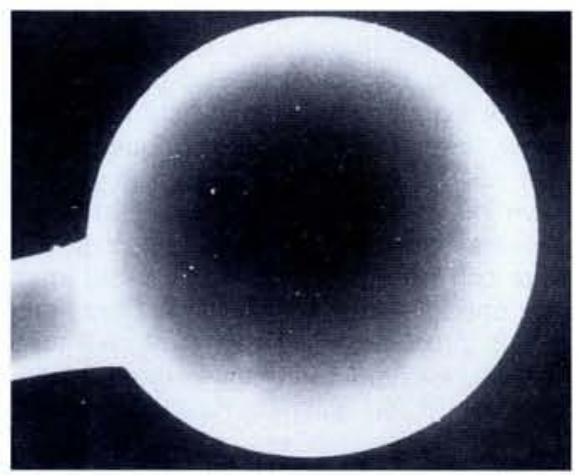

Fig. 1 - Electron microscope image of a $60 \mu \mathrm{m}$ diameter microsphere resonator for future atom-cavity studies. The sphere is obtained by melting the end of a high transmission glass fibre (seen at left) using a $\mathrm{CO}_{2}$ laser. The low-loss whispering gallery mode is localised along the equatorial plane of the sphere normal to the glass fibre stem.

binding one which is the difference combination of these two states. The former has an energy slightly lower than the sum energy of the separated atom and cavity subsystems, the latter an energy slightly larger. The binding energy of this "atom-cavity molecule" is typically of the order of a few nano-electron volts, about a factor of $10^{9}$ times smaller than that of an ordinary molecule. The atom-cavity interaction, which corresponds to microkelvin temperatures, is also very small compared to the usual kinetic energies of atoms.

\section{Experimental Observations}

These "atom-cavity" states have been recently observed in spectroscopy experiments. Researchers at the California Institute of Technology directed a laser beam across an optical cavity made of two closely spaced mirrors while a tenuous beam of caesium atoms was crossing the cavity [3]. The atoms and the cavity were tuned in exact resonance with each other and the laser frequency was scanned as its transmission across the cavity was recorded. When the cavity contained one atom on average, the transmission profile exhibited a double-peak structure, with an approximately $6 \mathrm{Mhz}$ splitting, one peak corresponding to the binding mode, the other to the antibinding mode of the compound atom-cavity system.

A similar experiment was performed at the Ecole Normale Supérieure (ENS) in Paris in which we observed a doublet with a splitting of about $100 \mathrm{kHz}$ associated with the exchange of a microwave photon between a superconducting niobium cavity and a few rubidium atoms prepared by laser excitation into a very excited Rydberg state [4]. In these experiments, the atoms were much too fast to allow for the formation of stable atom-cavity "molecules". The doublepeak spectra nevertheless revealed the transient existence of bound and antibound configurations during the time the atoms spent in the cavity.

\section{Novel resonators}

These experiments required the use of very small resonators because the strength of the atom-field coupling is inversely proportional to the square-root of the resonator's volume. Moreover, the photon must survive in the cavity for a time at least of the order of the period of energy exchange between the atom and the field mode. Two types of resonators have been used in experiments so far. For the microwave domain there are niobium superconducting cavities, often shaped in the form of centimetre-sized cylindrical boxes (cover illustration), which can provide storage times of up to a fraction of a second [5]. These cavities have to be coupled to very excited Rydberg atoms, which radiate photons in the microwave part of the spectrum. In the optical domain, Fabry-Perot resonators made of highly reflecting spherical mirrors with a multi-dielectric coating, facing each other at a millimetre distance, have photon damping times in the microsecond range.

Microsphere resonators of about $100 \mu \mathrm{m}$ diameter, made of highly transparent fused silica [6], are also very promising for future optical atom-cavity force studies (Fig. 1). These microspheres can sustain very weakly damped "whispering gallery modes" in which the field runs around the surface along a thin ring with transverse dimensions of the order of an optical wavelength. We have recently measured on these microspheres photon damping times of the order of one microsecond [7]. An atom located

\section{EPS LECTURER}

Professor Serge Haroche has agreed to be the EPS Lecturer for 1993/4. Starting in October 1993, he will be speaking at a number of centres throughout Europe on cavity quantum electrodynamics and other topics in atomic physics and quantum optics. 
close to the surface of the sphere should couple to the whispering gallery mode with a characteristic frequency in the $10-100 \mathrm{MHz}$ range. Strong atom-field correlations can thus build up during the photon storage time.

\section{Inverse Stern-Gerlach Experiment}

Adding photons to the cavity to enhance the tiny atom-cavity forces can be done by weakly coupling the cavity to an external radiation source. The atom-cavity exchange process is indeed stimulated by additional photons, its rate increasing linearly with the field amplitude. Photons introduced into the cavity add discrete quanta of energy to the antibinding state and subtract quanta of energy to the binding one, modifying accordingly the force felt by an atom in the cavity. This effect could be exploited to develop new, non-destructive ways to count small numbers of photons stored in a cavity. Many related schemes have been discussed recently as Gedanken experiments, and we are now trying at the ENS to reduce theory to practice.

In the simplest possible version [8-9], one would merely send one atom with a known velocity across the cavity and observe its trajectory (see Fig. 2). The atom will follow a different trajectory for each possible photon number. The detection of the atom, pinning down the trajectory, would reduce the field in the cavity into a well-defined photon number state. This is essentially analogous to the classic Stern-Gerlach experiment in which trajectories of particles passing through an inhomogeneous magnetic field are quantized according to their spin states. Here, the cavity is the Stern-Gerlach magnet and the observation of the atomic path yields information, not on the particle spin, but on the "magnet" internal states. For this reason, we have proposed calling this an "inverse Stern-Gerlach" experiment [8]. If the atom is sent along a symmetry axis of the cavity mode, the force is co-linear to the trajectory and the mechanical ef fects merely amount to a delay or an advan$\mathrm{ce}$ of the atom's propagation, whose measurement again yields the photon number in the cavity [9].

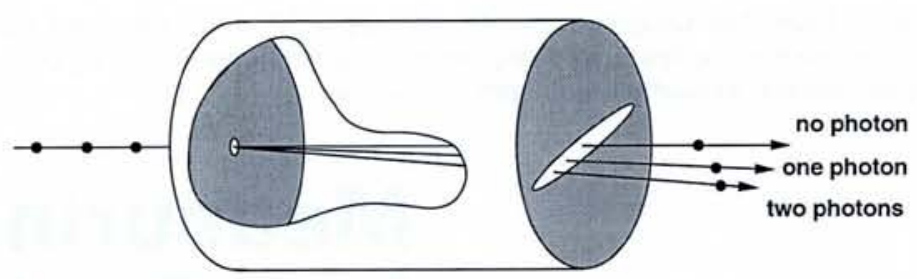

Fig. 2 - Schematic illustration of an inverse Stern-Gerlach experiment. An atom crossing the cavity is deflected into a trajectory which depends on the number of photons in the cavity.

In order to avoid an ambiguity in the assignment of a photon number to each trajectory, the atom-cavity system should be prepared in a pure binding or antibinding state, and not in a mixture of both. Such a preparation can be achieved by detuning slightly the atomic transition from the cavity mode. If the atomic transition frequency is a little short of cavity resonance, an excited atom in the presence of a given number of photons has slightly less energy than the state corresponding to the de-excited atom with one photon more. As an excited atom moves into the cavity, the system will then naturally evolve into the lower energy binding state. If the detuning has the opposite sign, the same trick will produce the antibinding state as well. These properties are reversed if the atom enters the cavity in its lower energy state (negative atom cavity detuning branches the system in the antibinding state in this case). These conclusions rely on the adiabatic theorem of quantum mechanics, which says that if a system's rate of evolution is slow enough, it follows continuously the state it is initially prepared in, provided the energy of this state never coincides with that of another one.

\section{Atomic Interferometry to Count Atoms}

A simple measurement of the atom's position at the cavity exit may not have enough resolution to distinguish small photon numbers from one another. Far more sensitive methods relying on atomic interferometry have been recently discussed [10-11]. Each atom in fact corresponds to a small wave packet with a de Broglie wavelength inversely proportional to its velocity. A 300 metre per second rubidium atom, for example, has a wavelength of $0.15 \AA$. The advance or retardation of the atom's motion means that its de Broglie wave is dephased by an angle depending on the photon number in the cavity. A delay equal to half the atom wavelength, a mere $0.075 \AA$, will result in the replacement of a crest of the matter wave by a trough, an effect which interferometric methods can easily demonstrate.

The method we have designed at the ENS [10], and are presently aiming to implement, is a variant of Ramsey fringe interferometry performed on Rydberg atoms (Fig. 3). It adds a few tricks to the simple scheme described above. Rydberg atoms are subjected to an auxiliary microwave pulse, which prepares them in a linear superposition of the upper and lower states of the atomic transition, just before entering the superconducting cavity containing the microwave field to be measured. When each atom enters the cavity, these states branch into the binding and antibinding atom-cavity states, respectively, so that the atom's wave function splits into an advanced part and a retarded part. On exiting the cavity, a second microwave pulse mixes again the upper and lower atomic states, in such a way as to construct the lower state, provided no phase lag occurred in the cavity. If the relative delay of the two parts of the atom wave function is equal to one-half the atom's de Broglie wavelength (or an odd number of it), the second microwave pulse reconstructs instead the upper atomic state. More generally, the probability of detecting either atomic state after the second microwave pulse appears as a periodic function

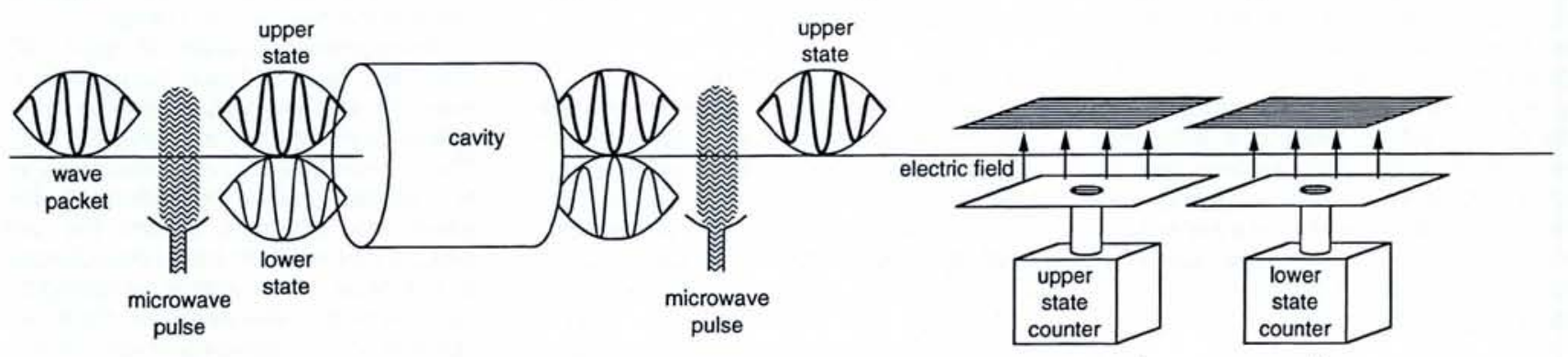

Fig. 3 - Sketch of the ENS atomic interferometry quantum non-demolition experiment. a, upper) The wavepacket of an atom, initially prepared in the upper state of the atomic transition, crosses the apparatus from left to right. The first microwave pulse changes it into a superposition of upper and lower state wavepackets, shown for clarity with different vertical offsets. The two parts of the wave function are delayed by different amounts in the cavity. In the situation illustrated here, the differential delay amounts to one-half the de Broglie wavelength. The second microwave pulse reconstructs in this case the upper atomic state. The atom state is analysed downstream with two field ionization detectors sensitive, repectively, to the upper and lower states of the transition. $b$, lower) The probabilities of detecting the atom in the upper and lower states exhibit interference fringes as a function of the number of photons in the cavity; these fringes are in phase opposition for the two states. Recording the fringe pattern by detecting a train of atoms amounts to a QND measurement of the photon number.

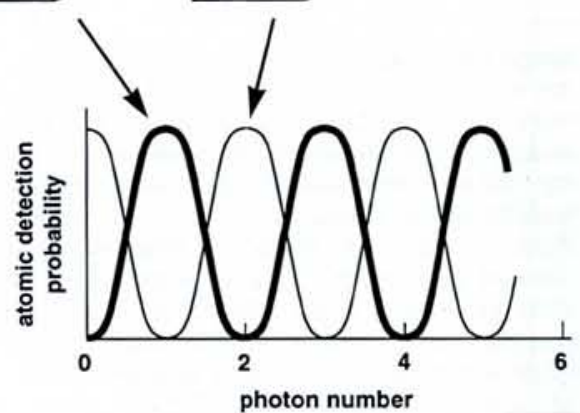


of the delay between the advanced and retarded parts of the atom's wave function.

Measuring the atom's final state thus provides information about the cavity-induced delay, and hence about the photon number in the cavity. State-selective detection of the atoms is performed by ionizing them in two small detectors and counting the resulting electrons. Each detector consists of plates with a small electric field across them: the first detector operates at a low field to ionize atoms in the upper level, and the second one at a higher field to ionize the lower level.

The radiation field fed into the cavity by a weak radiation source usually presents relatively large photon number fluctuations of an inherently quantum nature. Prior to measurement, the cavity thus contains a field described by a quantum photon number "wave function". The squared modulus of each wave function's component is the probability that the cavity stores a given photon number. Computer simulations show that this wave function progressively collapses into a pure photon number state, known as a Fock state, as successive atoms cross the cavity and are detected. If the experiment is resumed, each time with the same initial field in the cavity, the statistical distribution of photon numbers will be retrieved by the histogram of individual measurements. In any given run, however, the photon number will remain constant after it has been pinned down.

\section{A Quantum Non-Demolition Method}

The above method, which relies on atomcavity force effects using a slightly detuned cavity, will realise a feat of observation known as quantum non-demolition (QND). The photons are indeed counted without being destroyed, as opposed to conventional photodetection methods based on photon to electron conversion. The atoms are strongly coupled to the cavity field while they are inside the resonator, but they decouple adiabatically as they exit the cavity, leaving the field energy unaffected. The atom-cavity "collision" is an "elastic" process and the atom carries away information about the photon number, which is not destructive for the field intensity. After the field has been reduced into a Fock state, it remains unperturbed by further measurements, which will all yield the same result. This is completely at variance with conventional photon counting, which continuously deplete photons from the measured field. The method has the potential to measure extremely weak fields down to the single photon or even cavity vacuum level. Spurious photon counts will be essentially due to the low noise level of the state-selective atomic ionization counters and should be unlikely. Such QND measurements would have a sensitivity unattainable by conventional detection methods in the microwave region.

Atom-cavity QND methods should allow one to monitor events impossible to observe otherwise. For example, if a single photon disappears in the cavity walls due to small losses, this quantum jump event would register as a change of the atomic states detection probabilities. In this way, the death of photons could be witnessed, so to speak, in real-time without affecting its natural rate by the measurement process itself. The creation of a single photon in the cavity could be monitored as well. Such exquisitely sensitive measurements, performed at the quantum level, may one day be used to detect tiny forces which slightly deform the cavity boundaries, inducing discrete changes to photon numbers. QND processes were first mentioned in the context of gravity wave detection experiments [12], which imply the monitoring of exceedingly small length changes of a gravitational antenna. It remains to be seen whether the QND methods described here, or some variations based on them, can be of help in these very challenging experiments.

[1] Haroche S., Cavity Quantum Electrodynamics in Fundamental Systems in Quantum Optics, Proc. Les Houches Summer School, Session LIII. Eds.: J. Dalibard, et al. (North Holland) 1992; Haroche S. \& Raimond J.-M., Scientific American, April 1993, p. 26.
[2] Haroche S., et al., Europhys. Lett. 14 (1991) 19; Englert B.G., et al., ibid. 14 (1991) 24.

[3] Thompson B.J., et al., Phys. Rev. Lett. 68 (1992) 1132.

[4] Bernardot F., et al., Europhys. Lett. 17 (1991) 33.

[5] Rempe G., et al., Phys. Rev. Lett. 64 (1990) 2783.

[6] Braginsky V.B., et al., Phys. Lett. A 137 (1989) 393

[7] Collot L., et al., to be published (1993).

[8] Haroche S., et al., in Atomic Physics 13, Proc. 13th Int. Conf. on Atomic Physics, Eds.: H. Walther et al. (1993); Haroche S. \& Raimond J.-M. in Cavity Quantum Electrodynamics, special issue of Adv. in Atomic and Molecular Physics, Ed.: P. Berman (Academic Press, New York) 1993.

[9] Ivanov D. \& Kennedy T.A.B., Phys. Rev. A 47 (1993) 566.

[10] Brune M., et al., Phys. Rev. Lett. 65 (1990) 976; Phys. Rev. A 45 (1992) 5193.

[11] Holland M.J., et al., Phys. Rev. Lett. 67 (1991) 1716.

[12] Caves C.M., et al., Rev. Mod. Phys. 52 (1980) 431

\title{
High-Energy $\mathbf{C}_{60}$ Beams Available
}

\author{
S. Della-Negra and Y. Le Beyec of the Institut de Physique Nucleaire, Orsay, \\ outline progress in upgrading facilities for producing high-energy beams of \\ cluster ions including fullerenes.
}

The interactions of ions with surfaces and solid material have been investigated using a large variety of projectiles, ranging from the commonly used monoatomic ions up to dust particles. Recent work in several laboratories has demonstrated enhancement, relative to the rate for individual ions, of secondary ion emission (SIE) from various targets under the impact of clusters of atoms. The bombardment of organic and inorganic materials by $\mathrm{keV}$ ion clusters has also shown that cluster projectiles eject complex secondary ions from the surfaces more efficiently than single ions (Fig. 1 from [1]).

The energies of the beams used to perform these experiments are in the $\mathrm{keV}$ to a few tens of keV range; the yield of secondary electrons is rising sharply with the energy of impact so it is interesting to increase the kinetic energy using clusters with high masses. There are today no facilities available capable of producing suitably heavy and fast projectiles.

The availability of high energy, heavy projectile beams will also open up new domains owing to the large amount of energy deposited in a small volume of solid. For example, the linear energy loss easily reaches $5 \mathrm{keV} / \AA$

Fig. 1 - The yield of molecular ions from a phenylalanine target bombarded with $\mathrm{Au}^{+}$and Au cluster projectiles [1]. Data are plotted as a function of the projectile energy per unit mass $(E / A)$, equivalent to the square of the projectile velocity, so that the desorption yields obtained with different gold cluster projectiles can be compared at the same velocity. The molecular ion yield increases by a factor 30 on going from $\mathrm{Au}^{3+}$ to $\mathrm{Au}^{5+}$, implying that a nonlinear effect arises during cluster impact in the $\mathrm{keV}$ range. Other complex projectiles lead to the same yield enhancement. which is larger than the maximum values obtained with atomic projectiles such as uranium at a few MeV per nucleon. Secondly. the simultaneous impact of several atoms on a small surface $\left(100 \AA^{2}\right)$ can induce coherent effects: phenomena which must be studied include energy loss and fragmentation of the projectile, the charge density of the solid, pressure pulses and shock waves, defect production in insulators, and physical modification of the surface.

For example, it is necessary to validate theoretical work at Orsay [2] showing that a

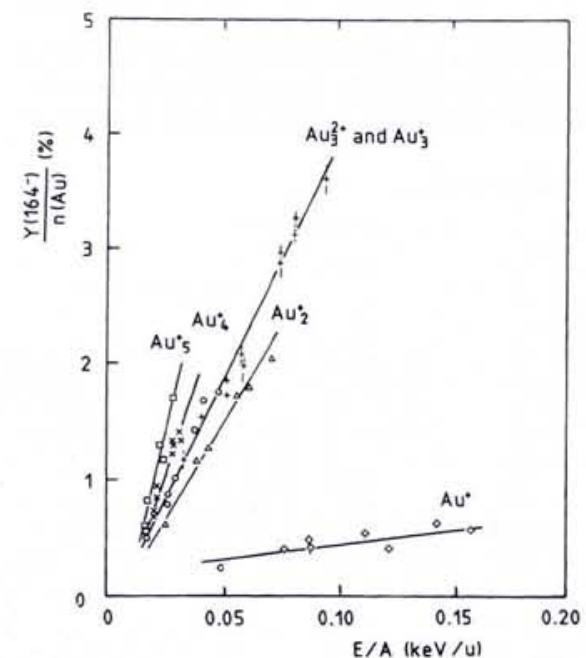

